\title{
El papel de la comunidad en la relación entre ciencia y fe. Aproximaciones a las propuestas epistemológicas de Thomas Kuhn y Mariano Artigas
}




\title{
El papel de la comunidad en la relación entre ciencia y fe. Aproximaciones a las propuestas epistemológicas de Thomas Kuhn y Mariano Artigas*
}

\begin{abstract}
Resumen: compartiendo un tiempo de búsqueda de verdades y la pasión por encontrarlas en sus investigaciones, Thomas Khun y Mariano Artigas, quizá por caminos que se entrecruzan, nos descubrirán un elemento clave: la comunidad humana. Es ella la que nos proporciona la estructura necesaria para que la ciencia y la fe encuentren caminos de resolución a las interrogantes del ser humano haciendo uso de las herramientas tan propias como lo es el lenguaje. Éste puede ser el camino privilegiado para encontrar alguna verdad que ayude a la humanidad a seguir comprendiéndose.
\end{abstract}

Palabras clave: ciencia, paradigma, comunidad, fe antropológica, lenguaje.

\section{The role of the community in the relationship between science and faith. Approaches to the epistemological proposals of Thomas Kuhn and Mariano Artigas}

\begin{abstract}
Sharing a time of searching for truths and the passion to find them in their researches, Thomas Khun and Mariano Artigas, perhaps by paths that intersect, will show us a key element: human community. It is what gives us the necessary structure so that science and faith may find ways of solving the questions of the human kind, making use of its own tools, such as language. This can be the privileged path to find some truth that helps humanity to continue to understand itself.
\end{abstract}

Keywords: science, paradigm, community, anthropological faith, language.

Fecha de recepción: 30 de abril de 2017

Fecha de aceptación: 7 de junio de 2017

Forma de citar (APA): Pereira Ríos, D. (2018). El papel de la comunidad en la relación entre ciencia y fe. Aproximaciones a las propuestas epistemológicas de Thomas Kuhn y Mariano Artigas. Revista Filosofía UIS, 17 (1), doi: ttp://dx.doi.org/10.18273/revfil. v17n1-2018013

Forma de citar (Harvard): Pereira Ríos, D. (2018). El papel de la comunidad en la relación entre ciencia y fe. Aproximaciones a las propuestas epistemológicas de Thomas Kuhn y Mariano Artigas. Revista Filosofía UIS, 17 (1), 271-287.

Diego Pereira Ríos: uruguayo. Estudiante del Profesorado de Filosofía (culminando), Universidad de Montevideo, Docente de Filosofía y Religión en Enseñanza Media.

Correo electrónico: pereira.arje@gmail.com

* Artículo de reflexión derivado de investigación. 


\section{El papel de la comunidad en la relación entre ciencia y fe. Aproximaciones a las propuestas epistemológicas de Thomas Kuhn y Mariano Artigas}

\section{Introducción}

A lo largo de la historia el ser humano se ha ocupado de investigar su accionar, a modo de un profundo estudio, imprescindible para lograr una mejor comprensión de sí mismo. A través de diversas disciplinas ha buscado dar respuestas a las interrogantes que se plantea $y$, aunque nada agota por completo su difícil definición, no siempre ha sido el centro de atención de su investigación. Pero esto no es una mirada negativa o acotadora sino que todo lo contrario es una posibilidad de que las investigaciones muestran que a lo largo de la historia se ha colocado al ser humano a veces en el centro, a veces en la frontera y otras veces por fuera.

Esta aclaración nos ayuda a comprender que en las disputas de las investigaciones de las ciencias humanas y las ciencias naturales no siempre se ha logrado la unificación de criterios sobre el ser humano. Dicho de otra manera, los estudios de la ciencia y de las humanidades no siempre coinciden en la mirada y el abordaje acerca del hombre. En una época donde vivimos el adiós a un cierto antropocentrismo que no se sostiene y donde algunos pensadores afirman la llegada de un nuevo geocentrismo, desde un nuevo paradigma ecológico, (Moltman-Boff, 2014, pp. 17-32), conviene a la reflexión filosófica un examen crítico y pausado de las diversas implicancias del ser humano a la luz de la historia de la ciencia y de los progresos científicos.

Este trabajo busca encontrar en la teoría epistemológica de Thomas Kuhn y en la propuesta de Mariano Artigas, un elemento común que nos dé razones para seguir creyendo en la ciencia como camino de realización humana pero, a su vez, hallar un nexo con la fe cristiana como dimensión trascendente y camino de plenitud. En 
esta oportunidad el elemento común está propuesto desde la comunidad humana que está en juego en una y otra dimensión, y que quizá pueda aportarnos algunas nuevas explicaciones. Estamos convencidos que en los dos ámbitos, el de la razón y el de la fe, el lugar de la comunidad que genera un ambiente plausible de desarrollo de la ciencia y la religión, es de primera importancia.

Confiamos en la unificación de los esfuerzos de los diferentes científicos y filósofos, teólogos y estudiosos del fenómeno religioso, para seguir buscando la unidad en la diversidad de propuestas actuales, que no siempre colaboran en la unión tan necesaria de la existencia humana, sino que la segregan. Creemos que es a partir de la comunidad, desde el pequeño grupo hasta la misma humanidad, que podemos generar nuevas formas de valoración de la vida a partir del mundo en el que vivimos. En este sentido hay algo en la esencia humana que la hace necesitada de la comunidad, no sólo por la transmisión de saberes de una generación a la otra, sino que tiene que ver con elementos que superan el ámbito lógico-racional -como la esperanza, la confianza, la fe misma- y que tienen que ver una dimensión metafísica, desde el ámbito de la razonabilidad y la afectividad.

Fe y razón, ciencia y religión, caminan juntas desde la aparición del ser humano sobre la tierra y han estado erróneamente enfrentadas - y en algunos casos hasta hoy - pero desde hace un tiempo se percibe un gran cambio. La unidad del cosmos hace que de la misma manera que el amor sigue siendo un misterio, también lo sea el mismo universo aún en sus grandes y exactas descripciones: "La ciencia que antes era un obstáculo, se ha convertido en una ayuda, una especie de trampolín hacia la espiritualidad y la mística [...] por otro lado, la religión, la espiritualidad y la mística se verán revolucionadas por esta nueva mentalidad científica" (Nolan, 2011, p. 76).

\section{Thomas Kuhn y Mariano Artigas}

Thomas Kuhn fue un judío norteamericano nacido en Cincinnati, Ohio, el 18 de julio de 1922 y fallecido en 1996, a los 73 años, en Massachusetts. En 1940 ingresa a Harvard comenzando sus estudios de física teórica. Forma parte de los epistemólogos post-popperianos que han desarrollado sus teorías con cierta base en la historia de la ciencia. Desde ella es que llega a afirmar que la investigación científica no se ha desarrollado según los modos hipotizados por Carnap (método inductivo-deductivo) y Popper (método hipotético-deductivo). Para él los textos elaborados en el trabajo científico implican una interpretación hermenéutica para una real comprensión de las teorías (Sánchez Campos, 2007). De ahí que, en esa lectura histórica que realiza acerca de la ciencia, encuentra que en muchos momentos el gran criterio de cientificidad lo da una noción sociológica: parte de un consenso (Klimovsky y De Asúa, 1992, p. 49), donde prima lo afectivo sobre lo estrictamente racional. Este sería un pacto entre los diferentes científicos que se ponen de acuerdo sobre ciertos problemas estudiados. 
Por su parte Mariano Artigas fue un autor español nacido en Zaragoza el 15 de diciembre de 1938. Fallece el 23 de diciembre de 2006 en Pamplona. Fue sacerdote católico y filósofo español que también dedicó esfuerzos al estudio dela ciencias físicas buscando vincular las verdades científicas y las verdades de la fe. Su actividad intelectual le dio la posibilidad de entrar en contacto con personalidades como Karl Popper, con quien mantuvo relación epistolar sobre diversas cuestiones filosóficas; con Evandro Agazzi y con el premio Nobel de medicina Sir John Ocles. En este vínculo de ciencia y fe centró todo su trabajo buscando una necesaria conciliación que quedó plasmada en sus libros, artículos y conferencias. Todo ello le mereció reconocimientos de gran nivel internacional.

\section{Los paradigmas: el desarrollo de la ciencia por revoluciones}

El libro La estructura de las revoluciones científicas contiene las ideas más originales de Kuhn. En ella reconstruye la historia del surgimiento de distintas disciplinas de la ciencia, en diversas etapas: la precientífica (o prehistórica), la etapa del logro individual de un científico, la conversión de los científicos al nuevo logro, el trabajo científico dentro de lo que llamará ciencia normal, la aparición de anomalías que a resolver, la crisis o emergencia que resquebraja el orden, la resolución del problema por parte de un científico, la nueva conversión al nuevo paradigma y por último la vuelta a la ciencia normal (Klimovsky y De Asúa, 1992, pp. 50-51). ¿Qué es un paradigma? Es el resultado de una investigación donde la comunidad científica reconoce la creación de un nuevo fundamento donde continuar su trabajo en el tiempo siguiente (D’Agostini, 2000, p. 495). El paradigma es lo que los científicos comparten como norma de trabajo y a su vez, es una comunidad que comparte un paradigma. Para Kuhn significa toda la constelación de creencias, valores, técnicas, etc. que comparten los miembros de una comunidad científica dada. Pero también denota las soluciones de problemas que son empleados como modelos y que pueden reemplazar reglas obligatorias dentro de la ciencia normal.

A su vez el paradigma es el conjunto de prácticas y categorías que permiten la organización del trabajo y ayuda a conocer el mundo de determinada manera, con lo cual el paradigma es un modelo de estudio científico donde los elementos que la integran dan funcionamiento a un modo de trabajo universalmente reconocido por la comunidad científica. De todos modos se da un momento donde aparecen las limitaciones y esto hace necesario un cambio del paradigma por uno nuevo. Para que suceda este cambio es necesaria una revolución que implica nuevas teorías y normas metodológicas. Se trata de una experiencia de quiebre con todos los conocimientos anteriores a nivel de la racionalidad de las estructuras como también de las funciones del pensamiento. 
La ciencia, según Kuhn, progresa pasando de tiempos de ciencia normal a cambios paradigmáticos, que son tiempos de ciencia extraordinaria, a partir de las revoluciones. En ellas un nuevo paradigma es sustituido por el nuevo, aceptado y puesto en práctica por la comunidad de científicos que ven la naturaleza tal como el paradigma se los representa. Esto implica una nueva definición del campo de manera mucho más rígida (Tomeo, 1966, p. 45). Al final de esa transición los profesionales de la ciencia habrán cambiado su visión del mundo logrando incluso un nuevo lenguaje para la comunicación. Esta traerá varios problemas. Uno de ellos versará sobre la inconmensurabilidad de los paradigmas: tienen un bagaje conceptual propio que no siempre es comprendido desde el lenguaje del paradigma anterior ni por los científicos que no pertenezcan a ese paradigma.

\section{Los límites de la ciencia y la posibilidad de la fe}

Uno de los temas necesarios y asumido en su trabajo por Artigas es el de la relación entre la ciencia y la religión, sobre todo desde la percepción general a partir de muchos cursos, talleres y seminarios que se dictan en varios lugares del mundo y en diferentes espectros (Artigas, 1999, pp. 611-639). Desde hace un buen tiempo muchos científicos, filósofos, teólogos y expertos del ámbito religioso vienen desarrollando un sano diálogo que busca conciliar la relación de oposición mantenida en un pasado. Lo podemos ver el caso del evolucionismo: para la ciencia a través del método experimental podemos conocer el progreso científico del universo ya que las ciencias naturales nos muestran con mesurada exactitud las dimensiones básicas de la naturaleza. Pero la ciencia deja entre paréntesis este tema dando posibilidad a la doctrina cristiana de la creación y por tanto a una posible acción divina que daría sentido a la realidad (Artigas, 2005).

Es una preocupación de Artigas poder encontrar los límites de la ciencia pues ve en ellos la posibilidad de una fe religiosa. Un gran obstáculo lo encuentra en el cientificismo "que es la afirmación de la ciencia como único modo válido de conocer la realidad, o como modelo que debe imitar toda pretensión de conocimiento verdadero" (Artigas, 2004, p. 125). Y este cientificismo es el que predomina aún en las mentalidades actuales, pero hoy de dos maneras: una optimista que ve a la ciencia como la única solución a todos los problemas humanos; y la pesimista que ve a la ciencia como la creadora de los grandes problemas de la historia, sobre todo desde el ejemplos de las bombas de Hiroshima y Nagasaki. La visión pesimista sobre le ciencia es extendida a todas las demás disciplinas que buscan la verdad: se asume que si la ciencia no puede resolver los enigmas del universo tampoco lo hará la filosofía o la religión.

Insiste este autor en tener claro que la ciencia es una actividad humana cuyo resultado es el conjunto de conocimientos a los cuales se llega por la aplicación del método científico. Dentro de ello los físicos buscan teorías de unificación que vinculen las fuerzas en el estado primitivo del universo y la comprensión de las 
leyes naturales de la actualidad. Esto implica un componente que cae por fuera del aspecto medible de la ciencia, y que supone ideas filosóficas sobre la realidad (Artigas, 2004, pp. 127-128). De este modo no hay nada en la física que pueda darle sentido al universo simplemente porque no puede, porque hablar del para qué del universo como una cuestión a futuro, sea temporal o aún en el plano metafísico, es cuestión de la filosofía o de la teología.

Uno de los errores de la ciencia positiva fue intentar darle a la ciencia el lugar ocupado por la religión como sucedió con Compte. El positivismo quiso ser una doctrina que respondiera a una actitud vital que está más allá de la lógica, como una concepción global del hombre y del mundo desde la visión científica. Desde el Círculo de Viena esta expresión se fundamenta en una filosofía que intentaba explicar y darle sentido a la vida humana. Compte buscaba la institución de una nueva religión universal con su doctrina, su moral e incluso su sacerdocio (Artigas, 2004, p. 135) donde la Razón sería la gran diosa. Hoy día esto está superado pero quedan los vestigios de un cientificismo como actitud vital propagada en los ámbitos académicos.

Debemos captar la diferencia que hay entre la existencia y la acción de Dios y la diferencia de los descubrimientos de la ciencia experimental, pues hablamos de planos diferentes. La ciencia tiene límites a partir de que no es un saber autónomo, autosuficiente, independiente de otros saberes. No es el último tribunal que puede juzgar la realidad. Sobre todo la ciencia experimental posee dos presupuestos que son filosóficos: el primero se trata de concepciones básicas acerca de la realidad, de la capacidad humana de conocerla; y el segundo la limitación de su estudio al plano netamente material, ya que hay realidades que escapan a la observación y la experimentación. Sin embargo debemos reconocer que esto no le da fiabilidad a la ciencia, sobre todo si examinamos algunas características: es intersubjetiva, pues los valores de la ciencia son comprobables por toda persona sin importar su filosofía o religión; es contrastable empíricamente pues sus enunciados se pueden poner a prueba experimentalmente; es predictible, pues puede predecir sucesos cuando se conocen sus antecedentes y, por último, es progresiva, lo que implica que existen criterios para distinguir cuando se realizan progresos auténticos (Artigas, 1992, pp. 53-54).

\section{La comunidad científica: punto de partida de la ciencia}

Siguiendo a Kuhn, uno de sus puntos claves es que para la aceptación de un paradigma es fundamental la comunidad científica. Es ella la que comparte las directrices de los posibles cambios. ¿Qué sería comunidad científica? Un grupo de científicos de varias disciplinas que han compartido aprendizajes, donde juntos entienden y resuelven los problemas de maneras muy similares y a través de un lenguaje común que hace posible su comunicación. Kuhn se detendrá 
en las creencias, los prejuicios y las filosofías experimentadas por los científicos destacando la importancia de las características sociológicas de las comunidades científicas.

Desde el interior de la comunidad encuentra el papel imprescindible de la relación pactada de sus miembros a la hora del desarrollo de la ciencia, incluyendo al científico nuevo o más joven. Estos acuerdos de aceptación, tanto de un paradigma o de un nuevo miembro del grupo, implica un consenso para la resolución de los diversos problemas. El cambio de un paradigma a otro es similar al proceso que se vive en el ámbito religioso pues implica un cambio de varios elementos del esquema mental e interior, similar a un cambio de Gestalt, que van Ilevando a los científicos a la adhesión al nuevo paradigma (Chalmers, 1982, p. 136).

Este comportamiento social de la comunidad científica tiene más elementos emocionales que racionales, pues son apuestas y ejercicios de confianza que superan el ámbito lógico. Aun así, las funciones formativas de los científicos implican prácticas, metodologías y comportamientos que identifican a cada miembro con su grupo. Desde los manuales de estudio, los problemas planteados y sus soluciones, todo ello revela las características de la comunidad. La resistencia al cambio de estos grupos humanos tiene que ver con su carácter conservador y dogmático, que no le permiten a los nuevos integrantes el aporte de algo novedoso. De aquí que si hablamos de subjetividad en la ciencia debemos pensar también, según Kuhn, en un sujeto plural —la comunidad de especialistas-que se encuentra en un determinado contexto histórico-cultural.

Esto derroca el mito del científico que trabaja de manera individual y que desde su laboratorio descubre las grandes verdades del mundo. Desde el ingreso a la comunidad científica el nuevo estudiante experimenta una especie de "adoctrinamiento" al ambiente donde deberá desarrollar su actividad, desde el cambio de intereses y prácticas hasta la experiencia de un aislamiento comunitario, frente a la sociedad, para dedicarse con más atención a resolver los problemas sobre los que tiene razones para creer que es capaz de resolver (Kuhn, 1971, p. 253). Esto no sólo convierte al iniciado en alguien especial sino que es toda la comunidad que lo siente así, y más aún: dentro de las creencias existe la idea de que el universo y la naturaleza poseen características especiales y es la comunidad la que tiene posibilidad de revelar sus misterios (266).

\section{La singularidad humana}

Para Mariano Artigas, en el posible puente de unión entre fe y ciencia, juega un papel imprescindible la singularidad humana y es por ello que desde los datos arrojados por la ciencia sobre el mismo ser humano desarrollará una importante reflexión sobre la persona. Desde las ciencias naturales el ser humano entra en un 
diálogo profundo con la naturaleza entablando con ella una posible relación que implican valores y creencias del orden racional, pero que también le da un estatuto en orden de lo sagrado, que obviamente transita por el camino de los sentimientos y la voluntad. Los procedimientos para el posible estudio e interrogación sobre la naturaleza son posibles por la singularidad humana, porque "los conceptos, leyes y teorías de la ciencia son construcciones de la mente humana" (Artigas, 1994).

Pero es claro que la persona no es solo cuerpo: es materia y es espíritu, que constituyen las dimensiones materiales y espirituales del género humano, unificados en una perfecta complejidad. De ahí que hay una dimensión de interioridad de la persona, en su misma unidad y que supera todo posible estudio científico, resguardando lo más íntimo y fundante del propio yo. Las ciencias humanas como la psicología, pueden acceder al caudal de datos históricos de cada sujeto para ayudarlo a interpretarlos, pero poco podrá decir del sentido de la vida. Cada persona valorar de diversos modos la vida y las circunstancias que atraviesa (Artigas, 1992, p. 174) y es esa misma autocomprensión que puede lograr toda persona, tiene consonancia con los descubrimientos científicos que posicionan al ser humano en un nivel superior en la escala de la naturaleza a nivel ontológico o cualitativo.

Esta dimensión del espíritu humano es la que posibilita la relación de la persona con Dios como ser trascendente o sobrenatural, que supera el ámbito de las percepciones empíricas y cognitivas. Dicho de otro modo, es el espíritu que hace posible la espiritualidad o experiencia de Dios. Recurriendo a la etimología de la palabra experiencia decimos que "es la ciencia o el conocimiento que el hombre adquiere cuando sale de sí mismo y estudia un objeto por todos lados" (Boff, 1975, pp. 22-25). En este sentido "ex" es un prefijo: salirse, hacia afuera; "peri" como raíz verbal, alrededor de, (empeiría), en torno de; y "encia", de ensente, como cualidad del agente. Por lo tanto la espiritualidad tiene que ver con el hacer experiencia de Dios en sentido de que la persona, al salirse de sus mismas posibilidades en búsqueda de un objeto capaz de ser alcanzado y conocido, interioriza una realidad que es superior a su misma naturaleza.

De este modo también podemos diferenciar la experiencia de lo que es la vivencia de la fe como situación psíquica causada en el espíritu con los sentimientos y emociones que afloran momentáneamente. Pero hay un elemento arrojado por Artigas que es importante: la evidencia alcanzada por la búsqueda de conocimiento. Esta evidencia puede ser directa o indirecta. La primera puede ser inmediata, o sea, alcanzada por sí mismo: la persona confirma algo sin más ayuda que sus propias capacidades a través de la observación o la intelección; o también puede ser mediata, por el uso del razonamiento. En este caso la evidencia es indirecta: está mediada por la palabra o conocimientos que producen actos, de otros a los que llamamos testigos. Casi la totalidad de nuestros conocimientos están basados en la evidencia indirecta como certezas de otros (Artigas, 1992, pp. 60-61). 
Desde esto -y en total consonancia con Kuhn- Artigas afirma la importancia de la dimensión sociológica de la ciencia a partir de la pregunta "iproporciona la ciencia experimental un conocimiento verdadero de la realidad, o se limita a proponer modelos más o menos útiles que no pueden considerarse propiamente como verdaderos?" (Artigas, 1992, p. 42). Estos modelos — los paradigmas— se basan en la confianza en la comunidad científica integrada por personas que dan testimonio de conocer ciertas verdades a las cuales el nuevo científico accede, desde la palabra y la aceptación racional de sus convicciones. Pero a esto se le suman elementos del orden ético: hay un consentimiento afectivo de adhesión al trabajo científico de otros en quien se cree.

\section{7. ¿Experiencia de fe antropológica?}

Es muy claro en la propuesta de Kuhn que la actividad científica requiere una gran dosis de fe, pero no sólo eso, sino que recurre a algunos conceptos del ámbito religioso para poder explicar las implicancias de los cambios paradigmáticos y de la experiencia vivida por el científico que ingresa a una comunidad. En este sentido es claro el recurso a las categorías sociológicas para dar cuenta de la importancia de la comunidad de científicos que está en juego. La comunidad posee cierta estructura que la lleva a mantener relaciones internas y externas que van configurando una cierta creencia acerca de la realidad. Esto trae consigo un compromiso del grupo y también la aceptación de un nuevo integrante.

Afirma Kuhn que si la aceptación de un nuevo candidato se juzga a partir de su relativa capacidad de resolver problemas se experimentarían muy pocas revoluciones (Kuhn, 1971, p. 244). Por lo tanto la aceptación de un nuevo miembro implica una cierta confianza anterior en el paradigma que guiará el trabajo. Pero este paradigma es ya un acuerdo entre la comunidad establecida, por lo que creer en el nuevo integrante, implica haber creído en toda la comunidad, por lo tanto es creer en hombres y mujeres. Esta fe, que llamaremos antropológica, es la que el mismo Kuhn afirma como fundamental en el proceso de aceptación de un nuevo paradigma. Quien lo acepta "deberá tener fe en que el nuevo paradigma tendrá éxito al enfrentarse a los muchos problemas que se presentan en su camino [...]" (245).

Pero centrándonos en el sujeto como criterio de orden sociológico que apunta a lo personal éste necesita de una base que — sin necesitar ser racional— sea de otro orden: la comunidad debe tener fe en el candidato particular que se escoja. Con esto demuestra que la explicación del desenvolvimiento de la ciencia es de un orden psicológico y sociológico pues todos los elementos que están en juego dependen de una aceptación y de un aval comunitario (De A. Dutra, 2007, pp. 93-120). Destacamos que en este proceso de ingreso a la comunidad hay un cierto pasaje de una razón entendida como facultad autónoma, no sólo aquella 
que puede penetrar en la realidad, sino que también combina la dimensión de libertad, de elección, del discernimiento; pero que poco a poco se va convirtiendo a las creencias del grupo (Catalão, 2007, pp. 678-681).

Por lo tanto creemos junto con Kuhn que hay una fe que no es solo implícita, sino que se manifiesta explícitamente en las relaciones de la comunidad científica. Desde la fenomenología apreciamos un cambio de conciencia similar a una conversión religiosa, que implica elementos que superan el ámbito racional donde la conciencia del candidato se une a la de la comunidad y es aceptado desde los testigos de la fe del sujeto en el paradigma al cual ellos pertenecen. Es una apuesta a una fe en el ser humano, no sólo en sus capacidades, sino que mantiene hasta una apuesta esperanzadora a los posibles logros que el sujeto y la comunidad pueden alcanzar. De aquí también decimos que hay una fe en el futuro de la ciencia desde las posibilidades humanas.

\section{8. ¿Religiosidad científica?}

Para responder esta pregunta primero necesitamos aclarar la noción de religiosidad, como expresión organizada de una cierta creencia de la cual se es integrante. Si hablamos de religión nos remitiremos a su sentido etimológico donde apreciamos dos nociones: el de re-legere (reunir, escoger, no descuidar) y el de re-ligare (atar, volver a ligar) (Queiruga, 1992, pp. 134-135). Y es a partir de aquí que captamos la idea de que hay algo que se busca, algo a lo cual unirse, pero en un sentido retroactivo: algo a lo cual ya se estuvo unido o vinculado en algún momento, un objeto del conocimiento que fue conocido y que en el estado actual se reconoce separado, lejano. Y porque es algo ya conocido tiene sentido el volver a tomar contacto nuevamente y cuidar de esa relación o vínculo. En este sentido tiene algo de la teoría de la reminiscencia de Platón.

Desde esta noción de religión tiene sentido hablar de ella tanto en la dimensión explícita de la fe religiosa como creencia en un ser trascendente, pero también podemos introducir este mismo sentido en la ciencia. Sea Dios en el ámbito religioso, sea la Verdad en la ciencia, ambos son buscados por una comunidad que se sostiene en un fundamento, que si bien tiene una cierta racionalidad, es sobre todo una fuerza sobrenatural: la fe. Esta fe parte de una constitución antropológica como búsqueda de trascender el ámbito de lo empírico para ir en búsqueda de una verdad que satisfaga las inquietudes y necesidades más profundas del ser humano.

El elemento clave de nuestra afirmación parte del lugar que Kuhn le da a la comunidad humana que hace posible la ciencia y que en el ámbito religioso también posibilita la fe como camino hacia Dios. En consonancia con la metafísica necesaria también en el ámbito filosófico el paradigma no sólo será una 
metodología de investigación, sino una manera de ver el mundo. En la Posdata al libro La estructura de las revoluciones científicas de 1969, Kuhn intentará aclarar su concepción cambiándola por matriz disciplinar. Esta nueva noción contiene algunos elementos que son compartidos en la comunidad científica, pero que también son elementos de una comunidad de fe.

Una matriz está compuesta por elementos diferentes pero ordenados de forma específica; y es disciplinar porque se refiere a la posesión común de los que practican una disciplina particular. Esta matriz disciplinar contiene varios elementos que afirman los elementos trascendentes de la comunidad y que posibilitan la fe: generalizaciones simbólicas que posibilitan la expresión de conceptos en palabras, modelos heurísticos que interpretan la realidad desde analogías y metáforas, ciertos valores que facilitan la unificación de la comunidad y que funcione como un todo completo. Este conjunto de elementos son parte de la formación que recibe el iniciado (Kuhn, 1971, pp. 280-286).

Los componentes de la experiencia de conversión de la comunidad científica se revelan a los sujetos como un fenómeno complejo suprarracional, a partir de una nota imprevisible que excede su comprensión. Todo esto se nos muestra como una verdad aceptada por revelación (Catalão, 2007, p. 681): la aceptación del nuevo paradigma genera una reestructura de las categorías mentales y procedimientos del científico, donde vive una experiencia fundante siempre a partir de la comunidad que atestigua dicho proceso de conversión. Pero este proceso es algo subjetivo, interior, que se sostiene en un el grupo humano como apoyo extrínseco (Dürr, 2007, p. 31), pero que dan fe que la nueva visión del mundo tiene que ver con todos. La aprobación del grupo da seguridad y sostén interior a las convicciones del nuevo sujeto.

\section{La complementariedad de la ciencia y la fe}

Desde la capacidad humana de la inteligencia admitimos que la naturaleza es posible de ser comprendida. Es lo que Artigas denomina inteligibilidad: sería un modo de ser captada la naturaleza por la inteligencia. Pero esta inteligencia tiene sus métodos propios de comprensión a partir de las diversas capacidades que posee: pensar, entender, comprender y diferenciar, tanto a nivel teórico como práctico. Desde esto, la comprensión de la naturaleza implica tener como objetivos el uso de la inteligencia en conjunción con la acción de la voluntad. Cada disciplina científica construye un objeto e intenta comprobar su validez desde factores convencionales (Artigas, 1992, pp. 305-317).

Pero es claro que dentro de estos factores convencionales están las demostraciones subjetivas e intersubjetivas que se generan en ciertos contextos, denotando la parcialidad de la realidad que depende del grupo, las búsquedas, 
las condiciones de posibilidad del tiempo, etc. Las construcciones humanas se suman a los procesos naturales de investigación y desde ello surge el conocimiento científico. En este sentido la inteligibilidad incluye aspectos prácticos que buscan la plenificación de la vida humana a través de la colaboración en la construcción de un mundo más humano. Esta apuesta creemos que es una apuesta de fe y confianza en el género humano y en la naturaleza como ámbito de desarrollo.

Ésta visión donde "la racionalidad científica resulta de una combinación peculiar de capacidades teóricas y prácticas" (Artigas, 1992, p. 57) no elimina la capacidad del ser humano del desarrollo de una espiritualidad que incluye la misma inteligencia y la voluntad de la persona y que busca trascender el ámbito de lo natural. Esto implica la aceptación libre de una acción metafísica fundante: la acción divina produce un ser que, sin dejar de pertenecer a la misma naturaleza, posee la capacidad de lo sobrenatural o trascendente (Artigas, 1992, p. 460). Pero esta misma necesidad es el presupuesto de que la fe es una aliado del conocimiento científico: no busca eliminarlo, por lo contrario le da un sentido de trascendencia a todo trabajo humano de investigación (Artigas, 2004, p. 150).

Todo lo que hemos afirmado, todo lo constatado e incluso lo dicho como apuestas a una conciliación entre fe y razón, ciencia y religión, tienen un fundamento: la capacidad humana de la inteligencia. Y la inteligencia no existe por sí misma sino que existe en el ser humano que, al dedicarse a la ciencia, forma parte de una comunidad que se estructura en función de elementos sobrenaturales que superan cualquier ecuación matemática o cálculo científico. Partiendo del humanismo, entendido éste como la preocupación más profunda del ser humano por el ser humano y el mundo, se hace necesario resaltar el papel del trabajo comunitario, en función de una convivencia cada vez más pacífica que logre la reunificación de todos los caminos de realización humana.

\section{Lenguaje y comunidad: el encuentro con la limitación}

Dando un último paso desde la importancia de la experiencia, a partir de la comunidad humana, creemos pertinente detenernos -al menos mínimamente- en la capacidad del ser humano de relacionarse y comunicarse, y desde allí comprenderse a partir de la posibilidad que le brinda el lenguaje. Un lenguaje que se transmite por símbolos y, dentro del lenguaje escrito, tendremos que el mismo Kuhn hablará en su desarrollo histórico de la ciencia por medio de metáforas. Sus obras están llenas de metáforas de las cuales tomaremos el caso del verbo ver: muchas veces se sentirá la ambigüedad de estar seguros de que Kuhn se refiere a un "ver con los ojos", como parte de la observación científica, o se trata de un "ver con la razón", como parte de la comprensión intelectual (De A. Dutra, 2007, p. 97). 
En este sentido se dan muchos errores por un mal uso de la razón, que como racionalidad tienen que ver con un error en la comprensión del lenguaje. Es lo que Kuhn refiere cuando hablará de la inconmensurabilidad: se trata de las redefiniciones de la ciencia que se revelan en el nuevo paradigma que hace que sus diferencias sean necesarias, pero a la vez se revelan como irreconciliables. De este modo Kuhn "afirma que el significado de una palabra depende del paradigma en el que esa misma se encuentre. Al cambiar el paradigma, cambian las relaciones entre las expresiones y, en consecuencia, el sentido de los términos" (Klimovsky y De Asúa, 1992, p. 52). De esta manera se entiende el problema del pasaje de un saber científico desde una comunidad a la otra, no sólo de los elementos objetivos, sino sobre todo de aquellos símbolos que intentan transmitir un cierto sentir acerca de la realidad y que no siempre se hace posible.

Ernst Cassirer, que presentaba al ser humano como ser simbólico, propuso desde la reflexión filosófica la delimitación del mundo objetivo, distinto del científico, donde aparecen a través del lenguaje grandes componentes del ámbito subjetivo que encierran siempre posibles críticas (Queiruga, 1992, p. 215). Pero dijimos que la inteligibilidad que posee la naturaleza hace posible al ser humano comprenderla, pero no en su totalidad, sino más bien como posibilidad o potencialidad (en términos metafísicos). Esto hace que cualquier explicación de la naturaleza siempre sea limitada, no sólo por el lenguaje humano -que no puede transmitir todo lo captable- sino que también por lo que la naturaleza se guarda para sí misma.

Dentro de esta discusión entra la polémica de las parábolas. Ellas simbolizan realidades que por analogía o semejanza, intentan cargar de sentido ciertas figuras que no se pueden detallar ni objetivizar. La afirmación "el universo es un misterio" implicaría un estudio desde el ámbito científico y desde el ámbito religioso para lograr dar una definición del término misterio. La ciencia irá por el camino de que misterioso es un objeto o hecho que no se puede describir o resolver, a modo de problema (Gabriel Marcel). La fenomenología de la religión dirá que un misterio es la manifestación de la divinidad o de lo trascendente en la creación, en un elemento natural (por ejemplo en la zarza ardiente que Moisés encontró en su camino¹). Por ello el lenguaje muchas veces sería el gran obstáculo de intercomprensión e interrelación entre las comunidades científicas e incluso las comunidades de fe.

\section{Conclusión: Ia comunidad como acceso a la Verdad}

Ciencia y fe son caminos seguros de acceso a la Verdad, orientadas cada una a su manera (Dürr, 2007, pp. 30-31). El científico buscará su verdad a partir de la investigación y desde el uso del método científico, pero muchas veces fragmentando tanto la realidad que le será muy difícil el encuentro con una única verdad. Por 
otro lado el creyente -o persona de fe, busca la verdad por medio de la dimensión religiosa como camino de acción y contemplación de un Dios que posee en sí la Verdad, pero de la cual sabe no se la puede apropiar por su misma naturaleza de criatura. Ambos son caminos no sólo válidos sino necesarios para un abordaje coherente de la realidad y como experiencias complementarias del ser humano. Uno desarrolla una actitud crítico-racional y la otra una contemplación-razonable.

Creemos que aún queda mucho camino por andar en la interpretación de los diversos lenguajes por el cual el ser humano se expresa y en los que la naturaleza se nos revela. Hombre-mundo-Dios, o criatura, creación y Creador, son inseparables a la hora de tomar cierta conciencia de las posibilidades de trascendencia, como de inmanencia, humana. En este sentido queremos recurrir al denominado último gran metafísico de la historia: Martin Heidegger. En sus escritos expresaba su pensamiento a través de frases tan metafóricas como paradigmáticas: "El lenguaje es la casa del ser. En su morada habita el hombre. Los pensadores y poetas son los guardianes de esa morada. Su guardia consiste en llevar a cabo la manifestación del ser, en la medida en que, mediante su decir, ellos la llevan al lenguaje y allí la custodian" (Heidegger, 1947, pp. 11-12).

Desde las diversas interpretaciones que podemos darle a esta frase y poniendo en juego algunos elementos de la hermenéutica como teoría del lenguaje, queremos afirmar la grandeza del lenguaje como un gran baúl que guarda el tesoro de la humanidad y que no se deja objetivar de una sola vez. Incluso este tesoro es custodiado justamente por aquellos que en sus descripciones apenas utilizan términos que se aproximan a la Verdad, pero que lejos están de desear describirla. Váttimo —que interpreta a Heidegger — dirá que "El lenguaje es esencialmente algo que disponemos y que sin embargo, en otra vertiente, dispone de nosotros, nos es entregado en cuanto lo hablamos, pero se apropia de nosotros [...]" (Váttimo, 2002, p. 114). Con ello el lenguaje muestra una vitalidad propia como la voz que trasciende al ser humano, el cual es instrumento que habla en nombre de alguien de quien no tenemos evidencia inmediata.

Si la ciencia buscara en esta frase un sentido puramente objetivo y demostrable le faltan los recursos para hacerlo. Si la filosofía del lenguaje lo intentara quizá podría ir un poco más allá y encontrar cierto sentido en ella. La metafísica aportaría sus descripciones del Ser en cuanto presente ontológicamente en todos los entes que existen en la realidad. Desde la religión también tendría sentido la identificación del Ser con Dios, desde una metafísica y una teología de la Encarnación: desde que Dios se hizo hombre en Jesús, Dios mismo se reveló al ser humano con palabras y obras concretas. Pero esa Palabra de Jesús es la misma Palabra de Dios que estaba en el principio de la Creación descripto en el libro del Génesis $(1,1)$ y que el evangelista Juan retoma en el inicio de su Evangelio: "Al principio existía la Palabra y la Palabra estaba junto a Dios, y la Palabra era Dios" (Jn 1, 1). 
En este sentido se reafirma la importancia de la comunidad humana, tanto en la ciencia como en la religión, ya que es desde ella que se hace posible la transmisión de un mensaje que viene pautado por un lenguaje que no siempre es factible de ser captado en su totalidad - y que quizá no deba serlo - pero que de todas maneras no indica que sea falso. Es la comunidad en sus relaciones y acuerdos internos que producen creencias y valores que la hacen promotoras de los nuevos descubrimientos, sean del orden objetivo-científico o del subjetivo-religioso, y que posibilitan la trascendencia temporal de sus convicciones. Por eso, toda comunidad humana posee en sí su verdad, que tiene relación directa con las búsquedas de todo el género humano, por lo que cada grupo deberá ser lo suficientemente abierto para poder realizar una red donde compartir sus saberes y creencias que posee como acceso a la verdad, y acceder a las verdades de los demás.

\section{Referencias}

Artigas, M. (1992). Ciencia y fe: nuevas perspectivas. Pamplona: EUNSA.

Artigas, M. (1995). La inteligibilidad de la naturaleza. Pamplona: EUNSA.

Artigas, M. (2004). Ciencia, razón y fe. Pamplona: EUNSA.

Boff, L. (1975). La experiencia de Dios. Bogotá: Stella.

Catalão, H. (2007). Thomas S. Kuhn no limiar da Filosofía da Religião. Revista Portuguesa de Filosofía, 63 (1-3), 677-681.

Chalmers, A. (1982). ¿Qué es esa cosa llamada ciencia? Una valoración de la naturaleza y el estatuto de la ciencia y sus métodos. España: Siglo Veintiuno Editores.

D' Agostini, F. (2000). Analíticos y continentales. Guía de la filosofía de los últimos treinta años. Madrid: Cátedra.

De A. Dutra, L. (2007). A Concepção Social da Investigação Científica segundo Thomas S. Kuhn. Revista Portuguesa de Filosofía, 63(1-3), 93-120.

De Laborda, A. (1989). La ciencia contemporánea y sus implicaciones filosóficas. Madrid: Cincel.

Dürr, H. (2007). Física e trascendência. Revista Portuguesa de Filosofía, 63(1-3), 25-47. 
Giberson, K. y Artigas, M. (2012). Oráculos de la ciencia. Científicos famosos contra Dios y la religión. Madrid: Encuentro.

Heidegger, M. (2006). Carta sobre el humanismo. Madrid: Alianza Editorial.

Klimovsky, G. y De Asúa, M. (1992). Corrientes epistemológicas contemporáneas. Buenos Aires: Centro Editor de América Latina.

Khun, T. (1971). La estructura de las revoluciones científicas. México: Fondo de Cultura Económica.

Moltman, J. y Boff, L. (2014). Há esperança para a criaçao ameaçada? Petrópolis: Vozes.

Nolan, A. (2011). Jesús. Hoy. Una espiritualidad de libertad radical. Santander: Sal Terrae.

Tomeo, A. (1996). Nosotros y la ciencia. Una introducción a la filosofía de la ciencia. Montevideo: Latina.

Torres Queiruga, A. (1992). La constitución moderna de la razón religiosa. Navarra: Verbo Divino.

Vattimo, G. (2002). Introducción a Heidegger. Barcelona: Gedisa.

\section{Cibergrafía}

Artigas, M. (1994). Ciencia y fe: nuevas perspectivas. Après Galilée. Science et foi: nouveau dialogue, sous la direction du cardinal Paul Paupard. Desclée de Brower, París, pp. 199-214. Recuperado de: http://www.unav.edu/web/ciencia-razon-yfe/ciencia-y-fe-nuevas-perspectivas

Artigas, M. (1999). El diálogo ciencia-fe en la Encíclica Fides et ratio. Anuario Filosófico, (32), 611-639. Recuperado de: http://www.unav.edu/web/cienciarazon-y-fe/el-dialogo-ciencia-fe-en-la-enciclica-fides-et-ratio

Artigas, M. (2005). Evolucionismo y fe cristiana. Seminario de CRYF, Universidad de Navarra. Recuperado de: http://www.unav.edu/web/ciencia-razon-y-fe/ evolucionismo-y-fe-cristiana 